Supplement of Biogeosciences, 14, 2133-2149, 2017

http://www.biogeosciences.net/14/2133/2017/

doi:10.5194/bg-14-2133-2017-supplement

(C) Author(s) 2017. CC Attribution 3.0 License.

(c) (i)

Supplement of

\title{
The oxic degradation of sedimentary organic matter 1400 Ma constrains atmospheric oxygen levels
}

Shuichang Zhang et al.

Correspondence to: Shuichang Zhang (sczhang@ petrochina.com.cn)

The copyright of individual parts of the supplement might differ from the CC-BY 3.0 licence. 
The oxic degradation of sedimentary organic matter 1.4 Ga constrains atmospheric oxygen levels

\section{Supplementary Information}

Table S1. Repeated analysis of standards by ICP-MS for V, Mo and U; by XRF for Al and Fe and by HHXRF for Fe.

\begin{tabular}{|c|c|c|c|c|c|c|c|c|c|c|c|}
\hline No & Sample & $\begin{array}{c}\mathrm{V} \\
(\mathrm{ppm})\end{array}$ & $\begin{array}{c}\text { Mo } \\
(\mathrm{ppm})\end{array}$ & $\begin{array}{c}\mathrm{U} \\
(\mathrm{ppm})\end{array}$ & No & Sample & $\begin{array}{c}\mathrm{Al} \\
(\mathrm{wt} \%)\end{array}$ & $\begin{array}{c}\mathrm{Fe} \\
(\mathrm{wt} \%)\end{array}$ & No & Sample & $\begin{array}{c}\text { HHXRF Fe } \\
(\mathrm{wt} \%)\end{array}$ \\
\hline 1 & GBW07309 & 99.6 & 0.62 & 2.48 & 1 & GBW07104 & 8.52 & 2.62 & 1 & PACS -3 & 4.08 \\
\hline 2 & GBW07309 & 98.4 & 0.63 & 2.51 & 2 & GBW07104 & 8.54 & 2.61 & 2 & PACS -3 & 4.07 \\
\hline 3 & GBW07309 & 93.3 & 0.67 & 2.57 & 3 & GBW07104 & 8.50 & 2.61 & 3 & PACS -3 & 3.99 \\
\hline 4 & GBW07309 & 93.7 & 0.62 & 2.68 & 4 & GBW07104 & 8.51 & 2.61 & 4 & PACS -3 & 4.07 \\
\hline 5 & GBW07309 & 99.9 & 0.61 & 2.50 & 5 & GBW07104 & 8.54 & 2.61 & 5 & PACS -3 & 3.93 \\
\hline 6 & GBW07309 & 94.7 & 0.66 & 2.70 & 6 & GBW07104 & 8.54 & 2.62 & 6 & PACS -3 & 3.95 \\
\hline 7 & GBW07309 & 96.5 & 0.67 & 2.48 & 7 & GBW07104 & 8.54 & 2.62 & 7 & PACS -3 & 3.98 \\
\hline 8 & GBW07309 & 95.3 & 0.61 & 2.57 & 8 & GBW07104 & 8.54 & 2.62 & 8 & PACS -3 & 3.96 \\
\hline 9 & GBW07309 & 93.9 & 0.64 & 2.56 & 9 & GBW07104 & 8.55 & 2.62 & 9 & PACS -3 & 3.95 \\
\hline \multirow[t]{5}{*}{10} & GBW07309 & 101.0 & 0.62 & 2.73 & 10 & GBW07104 & 8.54 & 2.63 & 10 & PACS -3 & 3.90 \\
\hline & & & & & 11 & GBW07104 & 8.54 & 2.61 & 11 & PACS -3 & 3.87 \\
\hline & & & & & & & & & 12 & PACS -3 & 3.95 \\
\hline & & & & & & & & & 13 & PACS -3 & 3.94 \\
\hline & & & & & & & & & 14 & PACS -3 & 3.95 \\
\hline average & & 96.6 & 0.64 & 2.58 & & & 8.53 & 2.62 & & & 3.97 \\
\hline std dev & & 2.9 & 0.02 & 0.09 & & & 0.02 & 0.01 & & & 0.06 \\
\hline Accepted & & 97.0 & 0.64 & 2.60 & & & 8.57 & 2.65 & & & 4.11 \\
\hline 1 & GBW07310 & 104.0 & 1.21 & 2.04 & 1 & GBW07407 & 15.51 & 10.14 & 1 & XML Z4 & 5.97 \\
\hline 2 & GBW07310 & 105.0 & 1.18 & 2.02 & 2 & GBW07407 & 15.48 & 10.14 & 2 & XML Z4 & 5.94 \\
\hline 3 & GBW07310 & 104.0 & 1.26 & 2.21 & 3 & GBW07407 & 15.45 & 10.12 & 3 & XML Z4 & 5.87 \\
\hline 4 & GBW07310 & 107.0 & 1.23 & 2.10 & & & & & 4 & XML Z4 & 5.90 \\
\hline
\end{tabular}




\begin{tabular}{|c|c|c|c|c|c|c|c|c|c|}
\hline 5 & GBW07310 & 110.0 & 1.24 & 2.07 & & & 5 & XML Z4 & 5.86 \\
\hline 6 & GBW07310 & 109.0 & 1.23 & 2.15 & & & 6 & XML Z4 & 5.87 \\
\hline 7 & GBW07310 & 107.0 & 1.19 & 2.12 & & & 7 & XML Z4 & 5.87 \\
\hline 8 & GBW07310 & 105.0 & 1.26 & 2.00 & & & 8 & XML Z4 & 5.92 \\
\hline 9 & GBW07310 & 109.0 & 1.16 & 2.14 & & & 9 & XML Z4 & 5.85 \\
\hline \multirow[t]{2}{*}{10} & GBW07310 & 106.0 & 1.17 & 2.09 & & & 10 & XML Z4 & 5.80 \\
\hline & & & & & & & 11 & XML Z4 & 5.91 \\
\hline average & & 106.6 & 1.21 & 2.09 & 15.48 & 10.13 & & & 5.89 \\
\hline std dev & & 2.2 & 0.04 & 0.06 & 0.03 & 0.01 & & & 0.05 \\
\hline Accepted & & 107.0 & 1.200 & 2.10 & 15.51 & 10.13 & & & 5.83 \\
\hline
\end{tabular}


Table S2. Geochemical data and sediment description for outcrop samples.

\begin{tabular}{|c|c|c|c|c|c|c|c|c|c|c|c|c|c|}
\hline Depth & Sediment & Org-C & $\delta^{13} \mathrm{C}$ & $\mathrm{Al}$ & $\mathrm{Fe}$ & $\mathrm{Fe} / \mathrm{Al}$ & $\mathrm{V}$ & $\mathrm{V} / \mathrm{Al}$ & Mo & $\mathrm{Mo} / \mathrm{Al}$ & $\mathrm{U}$ & U/Al & HI \\
\hline $\mathrm{M}$ & & $\mathrm{wt} \%$ & $\%$ & $\mathrm{wt} \%$ & $\mathrm{wt} \%$ & & $\mathrm{ppm}$ & & $\mathrm{ppm}$ & & $\mathrm{ppm}$ & & $\mathrm{mg} / \mathrm{gTOC}$ \\
\hline 0 & black & 1.14 & -34.1 & 5.77 & 5.39 & 0.93 & 80 & 13.9 & 1.54 & 0.27 & 2.6 & 0.45 & 138 \\
\hline 3.60 & green & 0.27 & -31.7 & 5.57 & 3.54 & 0.64 & 60 & 10.8 & 0.62 & 0.11 & 2.0 & 0.37 & 111 \\
\hline 4.60 & gray & 0.20 & -32.9 & 6.76 & 2.95 & 0.44 & 74 & 10.9 & 0.67 & 0.10 & 2.8 & 0.41 & 100 \\
\hline 6.10 & black & 2.25 & & 6.90 & 3.26 & 0.47 & 112 & 16.2 & 2.2 & 0.32 & 3.0 & 0.43 & 318 \\
\hline 7.60 & black & 3.32 & -33.4 & 6.95 & 3.31 & 0.48 & 163 & 23.4 & 5.28 & 0.76 & 4.0 & 0.58 & 373 \\
\hline 9.10 & black & 2.65 & & 6.73 & 3.42 & 0.51 & 203 & 30.2 & 4.63 & 0.69 & 3.8 & 0.56 & 353 \\
\hline 10.10 & black & 2.21 & -33.4 & 6.72 & 3.27 & 0.49 & 126 & 18.8 & 3.32 & 0.49 & 3.1 & 0.46 & 313 \\
\hline 11.10 & gray & 0.09 & -33.3 & 7.80 & 2.03 & 0.26 & 86 & 11.0 & 1.06 & 0.14 & 2.9 & 0.38 & 67 \\
\hline 12.10 & black & 3.37 & -32.8 & 7.57 & 3.22 & 0.43 & 221 & 29.2 & 5.48 & 0.72 & 4.7 & 0.63 & 353 \\
\hline 14.10 & gray & 0.94 & & 4.54 & 1.95 & 0.43 & 44 & 9.8 & 1.21 & 0.27 & 2.0 & 0.44 & 220 \\
\hline 14.60 & black & 3.86 & -33.0 & 9.06 & 2.28 & 0.25 & 239 & 26.4 & 3.25 & 0.36 & 4.9 & 0.54 & 338 \\
\hline 15.60 & gray & 0.23 & -32.7 & 10.10 & 2.22 & 0.22 & 132 & 13.1 & 0.83 & 0.08 & 4.0 & 0.40 & 43 \\
\hline 15.80 & gray & 0.20 & & 10.09 & 2.57 & 0.25 & 109 & 10.8 & 0.91 & 0.09 & 3.6 & 0.36 & 55 \\
\hline 17.10 & gray & 0.26 & -32.3 & 9.49 & 2.07 & 0.22 & 114 & 12.0 & 1.29 & 0.14 & 4.4 & 0.46 & 62 \\
\hline 18.60 & gray & 0.24 & & 9.20 & 2.95 & 0.32 & 89 & 9.7 & 1.88 & 0.20 & 3.6 & 0.39 & 71 \\
\hline 20.10 & gray & 0.18 & & 9.54 & 2.73 & 0.29 & 112 & 11.7 & 1.33 & 0.14 & 3.4 & 0.36 & 72 \\
\hline 21.60 & black & 3.92 & -32.4 & 8.18 & 4.20 & 0.51 & 245 & 29.9 & 7.22 & 0.88 & 5.0 & 0.61 & 319 \\
\hline 22.50 & gray & 0.30 & -32.2 & 9.35 & 2.62 & 0.28 & 106 & 11.3 & 1.46 & 0.16 & 3.9 & 0.41 & 93 \\
\hline 22.60 & gray & 0.15 & -31.6 & 9.07 & 2.62 & 0.29 & 103 & 11.4 & 1.83 & 0.20 & 3.8 & 0.42 & 80 \\
\hline 26.10 & black & 3.53 & & 7.64 & 4.53 & 0.59 & 223 & 29.2 & 5.34 & 0.70 & 4.6 & 0.60 & 310 \\
\hline 26.60 & gray & 0.40 & -32.0 & 8.60 & 2.73 & 0.32 & 108 & 12.6 & 2.22 & 0.26 & 4.1 & 0.47 & 113 \\
\hline 27.60 & black & 3.64 & -32.7 & 8.27 & 3.64 & 0.44 & 200 & 24.2 & 4.48 & 0.54 & 5.6 & 0.68 & 336 \\
\hline 28.40 & gray & 0.43 & & 8.65 & 2.50 & 0.29 & 119 & 13.7 & 1.94 & 0.22 & 4.6 & 0.53 & 128 \\
\hline 28.50 & black & 3.91 & -32.2 & 7.76 & 4.18 & 0.54 & 259 & 33.4 & 7.72 & 0.99 & 6.6 & 0.85 & 287 \\
\hline 29.00 & gray & 0.24 & & 8.78 & 1.90 & 0.22 & 105 & 12.0 & 1.03 & 0.12 & 3.7 & 0.42 & 104 \\
\hline 29.10 & black & 3.28 & & 8.15 & 3.85 & 0.47 & 271 & 33.2 & 7.04 & 0.86 & 6.3 & 0.78 & 241 \\
\hline 29.50 & gray & 0.30 & & 9.09 & 2.15 & 0.24 & 112 & 12.3 & 1.55 & 0.17 & 4.4 & 0.48 & 107 \\
\hline
\end{tabular}




$\begin{array}{lccccccccccccc}29.60 & \text { black } & 2.41 & & 7.81 & 3.56 & 0.46 & 132 & 16.9 & 4.36 & 0.56 & 5.3 & 0.67 & 256 \\ 30.10 & \text { gray } & 0.19 & -32.8 & 9.29 & 1.72 & 0.19 & 100 & 10.8 & 0.62 & 0.07 & 3.4 & 0.37 & 89 \\ 31.10 & \text { black } & 2.84 & & 8.07 & 4.19 & 0.52 & 169 & 20.9 & 4 & 0.50 & 5.9 & 0.72 & 205 \\ 34.10 & \text { black } & 3.02 & & 7.96 & 3.70 & 0.47 & 148 & 18.6 & 3.91 & 0.49 & 4.3 & 0.54 & 343 \\ 35.60 & \text { gray } & 0.35 & -32.5 & 8.36 & 1.49 & 0.18 & 110 & 13.2 & 1.07 & 0.13 & 3.2 & 0.39 & 120 \\ 36.60 & \text { gray } & 0.53 & & 8.34 & 1.63 & 0.20 & 114 & 13.7 & 1.29 & 0.15 & 3.4 & 0.40 & 160 \\ 37.60 & \text { black } & 3.42 & -33.0 & 7.93 & 2.08 & 0.26 & 216 & 27.2 & 5.5 & 0.69 & 5.2 & 0.66 & 430 \\ 39.00 & \text { gray } & 0.30 & -32.7 & 8.71 & 1.84 & 0.21 & 122 & 14.0 & 0.89 & 0.10 & 4.1 & 0.47 & 127 \\ 39.10 & \text { gray } & 0.19 & -31.7 & 8.37 & 1.72 & 0.20 & 77 & 9.2 & 0.66 & 0.08 & 3.1 & 0.37 & 100 \\ 40.60 & \text { gray } & 0.15 & & 8.11 & 1.93 & 0.24 & 80 & 9.9 & 0.68 & 0.08 & 3.0 & 0.37 & 73 \\ 42.10 & \text { gray } & 0.26 & -32.4 & 8.14 & 2.52 & 0.31 & 114 & 14.0 & 1.33 & 0.16 & 4.3 & 0.53 & 135 \\ 43.70 & \text { black } & 2.51 & -33.7 & 6.70 & 4.32 & 0.64 & 197 & 29.4 & 7.93 & 1.18 & 4.8 & 0.71 & 314 \\ 43.75 & \text { gray } & 0.28 & & 7.85 & 2.33 & 0.30 & 101 & 12.9 & 1.17 & 0.15 & 3.8 & 0.49 & 139 \\ 43.80 & \text { black } & 3.68 & -32.9 & 7.39 & 3.71 & 0.50 & 189 & 25.6 & 5.58 & 0.75 & 3.9 & 0.53 & 390 \\ 43.90 & \text { gray } & 0.31 & -31.9 & 7.55 & 1.82 & 0.24 & 104 & 13.8 & 1.07 & 0.14 & 4.1 & 0.54 & 145 \\ 43.94 & \text { black } & 3.10 & & 7.40 & 2.46 & 0.33 & 176 & 23.8 & 5.21 & 0.70 & 5.3 & 0.72 & 342 \\ 43.95 & \text { gray } & 0.25 & & 7.30 & 1.31 & 0.18 & 93 & 12.8 & 1.06 & 0.15 & 3.8 & 0.52 & 136 \\ 44.00 & \text { black } & 3.17 & & 7.09 & 4.09 & 0.58 & 249 & 35.1 & 10 & 1.41 & 5.6 & 0.78 & 302 \\ 44.10 & \text { black } & 3.51 & -33.3 & 7.03 & 3.21 & 0.46 & 246 & 35.0 & 7.65 & 1.09 & 6.0 & 0.85 & 403 \\ 44.40 & \text { black } & 3.40 & -33.6 & 7.04 & 3.56 & 0.51 & 238 & 33.8 & 7.58 & 1.08 & 6.2 & 0.88 & 385 \\ 44.70 & \text { black } & 2.48 & -33.8 & 6.69 & 3.53 & 0.53 & 178 & 26.6 & 6.75 & 1.01 & 4.7 & 0.70 & 363\end{array}$


Table S3. Fe speciation results from fresh core material

\begin{tabular}{cccccccccc}
\hline Depth & TOC & FeT & FeCARB & FeOX & FeMAG & FePy & FePy/FeHR & FeHR/FeT & $\begin{array}{c}\text { FeT } \\
\text { method }\end{array}$ \\
\hline M & wt\% & wt $\%$ & wt\% & wt $\%$ & wt $\%$ & wt $\%$ & & & \\
\hline 15.00 & 2.14 & 3.72 & 0.37 & 0.19 & 0.24 & 0.41 & 0.34 & 0.33 & HCl \\
15.71 & 0.21 & 2.71 & 0.05 & 0.16 & 0.03 & 0.19 & 0.44 & 0.16 & HCl \\
16.66 & 0.31 & 4.31 & 0.19 & 0.05 & 0.17 & 0.51 & 0.55 & 0.22 & HCl \\
17.85 & 0.36 & 4.13 & 0.34 & 0.05 & 0.10 & 0.77 & 0.61 & 0.30 & XRF \\
18.33 & 0.31 & 3.22 & 0.22 & 0.11 & 0.13 & 0.50 & 0.52 & 0.30 & XRF \\
18.8 & 0.39 & 4.71 & 0.47 & 0.09 & 0.23 & 0.78 & 0.49 & 0.33 & XRF \\
19.21 & 3.55 & 2.50 & 0.35 & 0.04 & 0.02 & 1.50 & 0.78 & 0.76 & HHXRF \\
19.75 & 0.41 & 4.14 & 0.47 & 0.06 & 0.14 & 0.72 & 0.52 & 0.34 & HCl \\
20.94 & 0.39 & 3.61 & 0.16 & 0.03 & 0.06 & 0.75 & 0.75 & 0.28 & HCl \\
21.89 & 0.53 & 4.00 & 0.24 & 0.03 & 0.09 & 0.97 & 0.73 & 0.33 & Wet \\
22.13 & 1.66 & 3.82 & 0.65 & 0.16 & 0.24 & 0.58 & 0.35 & 0.43 & XRF \\
22.84 & 0.38 & 3.51 & 0.15 & 0.03 & 0.06 & 0.71 & 0.74 & 0.27 & HCl \\
23.79 & 0.26 & 3.98 & 0.41 & 0.06 & 0.22 & 0.36 & 0.34 & 0.27 & HCl \\
24.74 & 0.18 & 4.62 & 0.24 & 0.05 & 0.26 & 0.33 & 0.38 & 0.19 & HCl \\
24.98 & 0.51 & 2.86 & 0.15 & 0.10 & 0.08 & 0.60 & 0.65 & 0.32 & XRF \\
25.46 & 3.92 & 5.60 & 1.26 & 0.15 & 0.31 & 1.46 & 0.46 & 0.57 & HHXRF \\
25.69 & 0.27 & 3.71 & 0.11 & 0.05 & 0.11 & 0.57 & 0.67 & 0.23 & HCl \\
25.93 & 0.79 & 5.65 & 0.18 & 0.04 & 0.15 & 1.09 & 0.74 & 0.26 & HHXRF \\
26.41 & 0.86 & 6.10 & 0.21 & 0.04 & 0.15 & 0.61 & 0.60 & 0.17 & HHXRF \\
26.64 & 0.29 & 3.64 & 0.20 & 0.05 & 0.09 & 0.83 & 0.71 & 0.32 & HCl \\
26.88 & 0.47 & 3.75 & 0.13 & 0.03 & 0.12 & 0.73 & 0.72 & 0.27 & HHXRF \\
27.36 & 0.93 & 5.35 & 0.14 & 0.04 & 0.12 & 0.52 & 0.64 & 0.15 & HHXRF \\
27.59 & 0.45 & 3.74 & 0.12 & 0.04 & 0.06 & 0.69 & 0.76 & 0.24 & HCl \\
27.83 & 0.47 & 2.88 & 0.08 & 0.06 & 0.03 & 0.73 & 0.81 & 0.31 & XRF \\
28.30 & 2.91 & 3.56 & 0.18 & 0.04 & 0.15 & 1.43 & 0.79 & 0.51 & HHXRF
\end{tabular}




\begin{tabular}{|c|c|c|c|c|c|c|c|c|c|}
\hline 28.54 & 0.99 & 3.58 & 0.17 & 0.04 & 0.09 & 1.08 & 0.78 & 0.39 & $\mathrm{HCl}$ \\
\hline 28.78 & 1.10 & 2.71 & 0.10 & 0.04 & 0.09 & 0.79 & 0.77 & 0.37 & HHXRF \\
\hline 29.26 & 1.02 & 3.07 & 0.23 & 0.08 & 0.14 & 1.23 & 0.74 & 0.54 & HHXRF \\
\hline 29.49 & 0.42 & 3.76 & 0.18 & 0.04 & 0.09 & 0.97 & 0.76 & 0.34 & $\mathrm{HCl}$ \\
\hline 29.73 & 0.67 & 2.85 & 0.21 & 0.05 & 0.12 & 0.79 & 0.68 & 0.41 & HHXRF \\
\hline 29.96 & 0.47 & 3.21 & 0.18 & 0.04 & 0.03 & 0.94 & 0.79 & 0.37 & $\mathrm{HCl}$ \\
\hline 30.21 & 0.51 & 2.68 & 0.08 & 0.04 & 0.09 & 0.68 & 0.76 & 0.34 & HHXRF \\
\hline 30.44 & 0.32 & 2.97 & 0.10 & 0.03 & 0.04 & 0.46 & 0.72 & 0.21 & $\mathrm{HCl}$ \\
\hline 30.68 & 2.52 & 3.72 & 0.70 & 0.11 & 0.10 & 0.63 & 0.41 & 0.42 & XRF \\
\hline 31.16 & 3.59 & 3.37 & 0.66 & 0.14 & 0.19 & 0.74 & 0.43 & 0.51 & HHXRF \\
\hline 31.39 & 0.43 & 3.33 & 0.30 & 0.04 & 0.08 & 0.93 & 0.69 & 0.41 & $\mathrm{HCl}$ \\
\hline 31.63 & 0.44 & 2.57 & 0.07 & 0.05 & 0.05 & 0.67 & 0.80 & 0.33 & HHXRF \\
\hline 32.11 & 1.08 & 3.41 & 0.27 & 0.05 & 0.09 & 1.38 & 0.77 & 0.53 & HHXRF \\
\hline 32.34 & 0.34 & 2.94 & 0.14 & 0.04 & 0.04 & 0.59 & 0.74 & 0.27 & $\mathrm{HCl}$ \\
\hline 32.58 & 0.71 & 2.84 & 0.12 & 0.05 & 0.05 & 1.11 & 0.84 & 0.47 & HHXRF \\
\hline 33.06 & 0.72 & 3.01 & 0.33 & 0.06 & 0.09 & 0.93 & 0.66 & 0.47 & HHXRF \\
\hline 33.29 & 0.87 & 4.88 & 0.37 & 0.05 & 0.08 & 2.21 & 0.82 & 0.56 & $\mathrm{HCl}$ \\
\hline 33.53 & 3.36 & 3.20 & 0.55 & 0.08 & 0.03 & 1.73 & 0.72 & 0.75 & $\mathrm{XRF}$ \\
\hline 34.01 & 3.41 & 3.48 & 0.46 & 0.07 & 0.07 & 2.03 & 0.77 & 0.75 & HHXRF \\
\hline 34.48 & 4.20 & 3.50 & 0.60 & 0.11 & 0.08 & 2.08 & 0.72 & 0.82 & HHXRF \\
\hline 34.96 & 3.50 & 3.39 & 0.59 & 0.09 & 0.12 & 1.82 & 0.69 & 0.77 & HHXRF \\
\hline 35.43 & 3.16 & 3.68 & 1.76 & 0.11 & 0.01 & 1.29 & 0.41 & 0.86 & HHXRF \\
\hline 35.91 & 4.77 & 3.06 & 0.40 & 0.06 & 0.04 & 1.78 & 0.78 & 0.74 & HHXRF \\
\hline 36.38 & 4.79 & 3.38 & 1.38 & 0.08 & 0.08 & 0.80 & 0.34 & 0.69 & XRF \\
\hline 36.86 & 3.95 & 3.04 & 0.43 & 0.07 & 0.04 & 1.80 & 0.77 & 0.77 & HHXRF \\
\hline 37.33 & 4.94 & 3.04 & 0.54 & 0.07 & 0.04 & 1.85 & 0.74 & 0.82 & HHXRF \\
\hline 37.81 & 4.01 & 3.00 & 0.40 & 0.06 & 0.04 & 1.80 & 0.78 & 0.77 & HHXRF \\
\hline 38.28 & 3.52 & 3.20 & 0.51 & 0.07 & 0.04 & 1.92 & 0.76 & 0.79 & HHXRF \\
\hline 38.76 & 3.11 & 2.90 & 0.49 & 0.07 & 0.06 & 1.40 & 0.69 & 0.70 & HHXRF \\
\hline
\end{tabular}




$\begin{array}{llllllllll}39.23 & 2.61 & 3.60 & 0.00 & 0.03 & 0.01 & 2.16 & 0.98 & 0.61 & \text { XRF } \\ 39.71 & 4.45 & 3.20 & 0.41 & 0.06 & 0.04 & 1.94 & 0.79 & 0.76 & \text { HHXRF } \\ 40.18 & 4.07 & 2.89 & 0.38 & 0.05 & 0.04 & 1.51 & 0.76 & 0.69 & \text { HHXRF } \\ 40.66 & 4.03 & 3.41 & 0.42 & 0.05 & 0.03 & 2.38 & 0.83 & 0.85 & \text { HHXRF } \\ 41.13 & 3.74 & 3.48 & 0.60 & 0.07 & 0.04 & 2.24 & 0.76 & 0.85 & \text { HHXRF } \\ 41.61 & 4.70 & 3.49 & 0.66 & 0.08 & 0.04 & 2.50 & 0.76 & 0.94 & \text { HHXRF } \\ 42.08 & 1.68 & 1.16 & 0.11 & 0.04 & 0.04 & 0.11 & 0.38 & 0.25 & \text { XRF } \\ 42.56 & 2.98 & 2.73 & 0.39 & 0.03 & 0.06 & 1.74 & 0.78 & 0.81 & \text { HHXRF } \\ 43.03 & 4.40 & 3.13 & 0.47 & 0.05 & 0.03 & 1.97 & 0.78 & 0.81 & \text { HHXRF } \\ 43.51 & 4.20 & 3.28 & 0.51 & 0.05 & 0.03 & 2.24 & 0.79 & 0.86 & \text { HHXRF } \\ 43.98 & 3.16 & 3.08 & 0.48 & 0.02 & 0.03 & 1.95 & 0.79 & 0.81 & \text { HHXRF } \\ 44.46 & 4.83 & 3.08 & 0.53 & 0.05 & 0.03 & 2.19 & 0.78 & 0.91 & \text { HHXRF } \\ 44.93 & 3.93 & 2.39 & 0.54 & 0.04 & 0.01 & 1.16 & 0.66 & 0.73 & \text { XRF }\end{array}$


Table S4. Parameters used to calculate $\mathrm{O}_{2}$ exposure time and OC burial efficiencies

\begin{tabular}{|c|c|c|c|c|c|c|c|c|}
\hline site & Depth & $\begin{array}{c}\mathrm{O}_{2} \\
\text { pen. }\end{array}$ & Sed rate & OrgC & $\begin{array}{c}\mathrm{O}_{2} \\
\text { uptake } \\
\text { rate }\end{array}$ & $\begin{array}{c}\mathrm{O}_{2} \\
\text { exposure } \\
\text { time }\end{array}$ & $\begin{array}{c}\text { Burial } \\
\text { eff. }\end{array}$ & ref \\
\hline & $\mathrm{m}$ & $\mathrm{cm}$ & $\mathrm{cm} \mathrm{y}^{-1}$ & $\mathrm{wt} \%$ & $\begin{array}{c}\mathrm{mmol} \mathrm{O}_{2} \\
\mathrm{~cm}^{-2} \mathrm{y}^{-1}\end{array}$ & years & $\%$ & \\
\hline Cape lookout Bight & 10 & 0.30 & $5.4 \times 10^{0}$ & 3.0 & $2.1 \times 10^{0}$ & $5.6 \times 10^{-2}$ & 83.0 & (Canfield, 1989) \\
\hline Aarhus Bay & 16 & 0.60 & $1.7 \times 10^{-1}$ & 2.0 & $6.5 \times 10^{-1}$ & $3.6 \times 10^{0}$ & 25.0 & (Jørgensen, 1996; Thamdrup et al., 1994) \\
\hline Kattegat/Skagerrak Sta 2 & 25 & 0.25 & $1.3 \times 10^{-1}$ & 1.5 & $6.3 \times 10^{-1}$ & $1.9 \times 10^{0}$ & 16.6 & (Jørgensen and Revsbech, 1989) \\
\hline Young Sound, Greenland & 36 & 1.00 & $1.2 \times 10^{-1}$ & 1.3 & $2.4 \times 10^{-1}$ & $8.5 \times 10^{0}$ & 29.6 & (Rysgaard et al., 1998) \\
\hline Katt/Skagerrak Sta 6 & 43 & 0.42 & $2.6 \times 10^{-1}$ & 1.4 & $3.9 \times 10^{-1}$ & $1.6 \times 10^{0}$ & 37.6 & (Jørgensen and Revsbech, 1989) \\
\hline Katt/Skagerrak Sta 9 & 65 & 0.55 & $2.6 \times 10^{-1}$ & 1.7 & $4.4 \times 10^{-1}$ & $2.1 \times 10^{0}$ & 39.0 & (Jørgensen and Revsbech, 1989) \\
\hline Katt/Skagerrak Sta 10 & 73 & 0.30 & $2.6 \times 10^{-1}$ & 1.8 & $8.3 \times 10^{-1}$ & $1.2 \times 10^{0}$ & 27.0 & (Jørgensen and Revsbech, 1989) \\
\hline Svalbard Sta 3 & 115 & 0.96 & $3.6 \times 10^{-1}$ & 1.8 & $4.8 \times 10^{-1}$ & $2.6 \times 10^{0}$ & 46.7 & (Glud et al., 1998; Sagemann et al., 1998) \\
\hline Svalbard Sta 2 & 155 & 0.55 & $6.1 \times 10^{-1}$ & 1.5 & $6.0 \times 10^{-1}$ & $9.0 \times 10^{-1}$ & 49.5 & (Glud et al., 1998; Sagemann et al., 1998) \\
\hline Svalbard Sta 5 & 175 & 1.10 & $1.8 \times 10^{-1}$ & 2.5 & $3.2 \times 10^{-1}$ & $6.0 \times 10^{0}$ & 47.7 & (Glud et al., 1998; Sagemann et al., 1998) \\
\hline Katt/Skagerrak Sta 12 & 200 & 0.32 & $2.6 \times 10^{-1}$ & 2.4 & $5.0 \times 10^{-1}$ & $1.2 \times 10^{0}$ & 44.4 & (Jørgensen and Revsbech, 1989) \\
\hline California slope Sta K & 1000 & 0.50 & $6.2 \times 10^{-3}$ & 3.9 & $4.3 \times 10^{-2}$ & $8.0 \times 10^{1}$ & 26.6 & (Reimers et al., 1992) \\
\hline Chilean coast Sta 40 & 1015 & 0.60 & $3.1 \times 10^{-2}$ & 2.0 & $2.1 \times 10^{-1}$ & $1.9 \times 10^{1}$ & 16.0 & (Thamdrup and Canfield, 1996) \\
\hline Northeast Atlantic Sta B & 1023 & 1.60 & $1.6 \times 10^{-3}$ & 0.3 & $5.4 \times 10^{-2}$ & $1.0 \times 10^{3}$ & 0.6 & (Lohse et al., 1998) \\
\hline California rise Sta D & 1440 & 1.00 & $1.0 \times 10^{-3}$ & 1.7 & $3.5 \times 10^{-2}$ & $9.6 \times 10^{2}$ & 3.1 & (Reimers et al., 1992) \\
\hline California rise Sta $\mathrm{L}$ & 1890 & 0.90 & $3.0 \times 10^{-3}$ & 1.2 & $5.3 \times 10^{-2}$ & $3.0 \times 10^{2}$ & 4.2 & (Reimers et al., 1992) \\
\hline San Clemente Basin & 1900 & 0.80 & $2.0 \times 10^{-2}$ & 2.0 & $4.5 \times 10^{-2}$ & $4.1 \times 10^{1}$ & 36.0 & (Bender et al., 1989) \\
\hline Chilean coast Sta 41 & 2000 & 0.60 & $3.5 \times 10^{-2}$ & 2.2 & $1.7 \times 10^{-1}$ & $1.7 \times 10^{1}$ & 20.9 & (Thamdrup and Canfield, 1996) \\
\hline Borderland Basins-SCI & 2053 & 0.70 & $2.0 \times 10^{-2}$ & 2.6 & $4.0 \times 10^{-2}$ & $3.6 \times 10^{1}$ & 45.0 & (Berelson et al., 1996; Reimers, 1987) \\
\hline California rise Sta $\mathrm{H}$ & 3580 & 1.50 & $3.6 \times 10^{-3}$ & 2.0 & $6.2 \times 10^{-2}$ & $4.1 \times 10^{2}$ & 7.0 & (Reimers et al., 1992) \\
\hline Borderland Basins-PE & 3707 & 2.00 & $3.9 \times 10^{-2}$ & 1.1 & $1.4 \times 10^{-2}$ & $5.1 \times 10^{2}$ & 17.0 & (Berelson et al., 1996) \\
\hline Northeast Atlantic Sta III & 3719 & 5.50 & $2.1 \times 10^{-3}$ & 0.4 & $5.7 \times 10^{-2}$ & $2.6 \times 10^{3}$ & 0.9 & (Lohse et al., 1998) \\
\hline California rise Sta $\mathrm{M}$ & 3730 & 1.60 & $1.3 \times 10^{-2}$ & 2.8 & $6.4 \times 10^{-2}$ & $1.2 \times 10^{2}$ & 26.7 & (Reimers, 1987; Reimers et al., 1992) \\
\hline Northeast Atlantic Sta E & 4486 & 10.00 & $2.3 \times 10^{-3}$ & 0.4 & $3.6 \times 10^{-2}$ & $4.3 \times 10^{3}$ & 1.6 & (Lohse et al., 1998) \\
\hline
\end{tabular}


Table S5. $\mathrm{O}_{2}$ penetration depths, bottom water $\mathrm{O}_{2}$ levels and sediment $\mathrm{O}_{2}$ uptake rates for modern sediments.

\begin{tabular}{|c|c|c|c|c|c|}
\hline Site & $\begin{array}{c}\text { Water } \\
\text { depth (m) }\end{array}$ & $\begin{array}{c}\mathrm{O}_{2} \text { pen. } \\
(\mathrm{cm})\end{array}$ & $\begin{array}{c}\mathrm{BW} \mathrm{O}_{2} \\
(\mu \mathrm{M})\end{array}$ & $\begin{array}{c}\mathrm{O}_{2} \text { uptake } \\
\mathrm{mmol}^{-2} \\
\mathrm{~cm}^{-2} \mathrm{y}^{-1}\end{array}$ & reference \\
\hline Cape lookout Bight & 10 & 0.30 & 250 & 2.050 & (Chanton et al., 1987) \\
\hline Western Pac sta I & 4765 & 60.00 & 250 & 0.005 & (Murray and Grundmanis, 1980) \\
\hline Western Pac Sta J & 5361 & 60.00 & 250 & 0.005 & (Murray and Grundmanis, 1980) \\
\hline Western Pac StaK & 5469 & 20.00 & 250 & 0.008 & (Murray and Grundmanis, 1980) \\
\hline Kattegat/Skagerrak Sta3 & 15 & 0.20 & 250 & 0.800 & (Jørgensen and Revsbech, 1989) \\
\hline Kattegat/Skagerrak Sta 8 & 17 & 0.45 & 250 & 0.550 & (Jørgensen and Revsbech, 1989) \\
\hline Kattegat/Skagerrak Sta 2 & 25 & 0.25 & 250 & 0.630 & (Jørgensen and Revsbech, 1989) \\
\hline Kattegat/Skagerrak Sta 6 & 43 & 0.42 & 250 & 0.390 & (Jørgensen and Revsbech, 1989) \\
\hline Kattegat/Skagerrak Sta 9 & 65 & 0.55 & 250 & 0.440 & (Jørgensen and Revsbech, 1989) \\
\hline Kattegat/Skagerrak Sta 10 & 73 & 0.30 & 250 & 0.830 & (Jørgensen and Revsbech, 1989) \\
\hline Kattegat/Skagerrak Sta 12 & 200 & 0.32 & 250 & 0.500 & (Jørgensen and Revsbech, 1989) \\
\hline Central Calif slope-rise & 791 & 0.40 & 250 & 0.037 & (Reimers et al., 1992) \\
\hline Skagerrak S4 & 190 & 0.70 & 250 & 0.580 & (Canfield et al., 1993) \\
\hline Skagerrak S9 & 695 & 1.60 & 250 & 0.400 & (Canfield et al., 1993) \\
\hline Chile sta 40 & 1015 & 0.60 & 250 & 0.210 & (Thamdrup and Canfield, 1996) \\
\hline Chile sta 41 & 2000 & 0.60 & 250 & 0.170 & (Thamdrup and Canfield, 1996) \\
\hline Svalbard Sta 3 & 115 & 0.96 & 250 & 0.480 & (Glud et al., 1998) \\
\hline Svalbard sta 4 & 138 & 0.93 & 250 & 0.440 & (Glud et al., 1998) \\
\hline Svalbard sta 5 & 175 & 1.10 & 250 & 0.320 & (Glud et al., 1998) \\
\hline Svalbard Sta 2 & 155 & 0.55 & 250 & 0.600 & (Glud et al., 1998) \\
\hline S. Atlantic Geob 1713 & 604 & 0.40 & 250 & 0.340 & (Glud et al., 1994) \\
\hline S. Atlantic Geob 1719 & 1015 & 1.20 & 250 & 0.410 & (Glud et al., 1994) \\
\hline S. Atlantic Geob 1703 & 1747 & 1.10 & 250 & 0.566 & (Glud et al., 1994) \\
\hline S. Atlantic Geob 1711 & 1947 & 2.50 & 250 & 0.110 & (Glud et al., 1994) \\
\hline S. Atlantic Geob 1721 & 3095 & 4.10 & 250 & 0.153 & (Glud et al., 1994) \\
\hline S. Atlantic Geob 1702 & 3107 & 3.50 & 250 & 0.066 & (Glud et al., 1994) \\
\hline
\end{tabular}




$\begin{array}{lcccc}\text { Aarhus Bay } & 16 & 0.60 & 250 & 0.650 \\ \text { Arctic ocean-19/050 } & 170 & 0.50 & 250 & 0.409 \\ \text { Arctic ocean-19/143 } & 188 & 0.60 & 250 & 0.143 \\ \text { Arctic ocean-19/134 } & 273 & 3.80 & 250 & 0.117 \\ \text { Arctic ocean-19/070 } & 318 & 0.50 & 250 & 0.335 \\ \text { Arctic ocean-19/082 } & 326 & 0.80 & 250 & 0.340 \\ \text { Arctic ocean-19/119 } & 486 & 2.50 & 250 & 0.092 \\ \text { Arctic ocean-19/101 } & 530 & 3.00 & 250 & 0.104 \\ \text { Arctic ocean-19/086 } & 550 & 1.70 & 250 & 0.210 \\ \text { Arctic ocean-19/100 } & 867 & 1.70 & 250 & 0.149 \\ \text { Arctic ocean-19/112 } & 1010 & 3.70 & 250 & 0.067 \\ \text { Arctic ocean-19/078 } & 2010 & 1.70 & 250 & 0.134 \\ \text { Arctic ocean-19/108 } & 2490 & 6.00 & 250 & 0.110 \\ \text { Arctic ocean-19/098 } & 2577 & 5.00 & 250 & 0.097 \\ \text { Amazon shelf RMT-1 } & 9 & 0.15 & 250 & 0.440 \\ \text { Amazon shelf OST-1 } & 15 & 0.50 & 250 & 0.400 \\ \text { Amazon shelf RMT-2 } & 18 & 0.15 & 250 & 0.580 \\ \text { Amazon shelf OST-2 } & 20 & 0.50 & 250 & 0.390 \\ \text { Amazon shelf OST-3 } & 40 & 0.15 & 250 & 0.470 \\ \text { Amazon shelf RMT-3 } & 44 & 0.45 & 250 & 0.580 \\ \text { Patton Escarpment, E.N. } & & & & \\ \text { PAC } & 3750 & 3.00 & 250 & 0.065 \\ \text { Young Sound, Greenland } & 36 & 1.00 & 250 & 0.235 \\ \text { JGOFS 02S } & 4300 & 5.00 & 250 & 0.020 \\ \text { JGOFS 02N } & 4400 & 6.00 & 250 & 0.019 \\ \text { JGOFS 05N } & 4500 & 10.00 & 250 & 0.011 \\ \text { JGOFS 00 } & & 6.00 & 250 & 0.018 \\ \text { Northeast Atlantic StaI } & 668 & 0.80 & 250 & 0.086 \\ \text { Northeast Atlantic StaB } & 1023 & 1.60 & 250 & 0.054 \\ \text { Northeast Atlantic StaII } & 1442 & 2.50 & 250 & 0.065 \\ \text { Northeast Atlantic StaC } & 1989 & 3.50 & 250 & 0.039\end{array}$

(Thamdrup et al., 1994)

(Hulth et al., 1994)

(Hulth et al., 1994)

(Hulth et al., 1994)

(Hulth et al., 1994)

(Hulth et al., 1994)

(Hulth et al., 1994)

(Hulth et al., 1994)

(Hulth et al., 1994)

(Hulth et al., 1994)

(Hulth et al., 1994)

(Hulth et al., 1994)

(Hulth et al., 1994)

(Hulth et al., 1994)

(Aller et al., 1996)

(Aller et al., 1996)

(Aller et al., 1996)

(Aller et al., 1996)

(Aller et al., 1996)

(Aller et al., 1996)

(Reimers et al., 1986; Reimers and Suess, 1983)

(Rysgaard et al., 1998)

(Hammond et al., 1996)

(Hammond et al., 1996)

(Hammond et al., 1996)

(Hammond et al., 1996)

(Lohse et al., 1998)

(Lohse et al., 1998)

(Lohse et al., 1998)

(Lohse et al., 1998) 


$\begin{array}{lcrrcc}\text { Northeast Atlantic Sta III } & 3719 & 5.50 & 250 & 0.057 & \text { (Lohse et al., 1998) } \\ \text { Northeast Atlantic StaE } & 4486 & 10.00 & 250 & 0.036 & \text { (Lohse et al., 1998) } \\ \text { Borderland Basins-PE } & 3707 & 2.00 & 131 & 0.014 & \text { (Berelson et al., 1996) } \\ \text { Central Calif Sta M } & 3730 & 1.60 & 129 & 0.064 & \text { (Reimers et al., 1992) } \\ \text { East N. Pac NH14 } & 114 & 0.40 & 127 & 0.670 & \text { (Devol and Christensen, 1993) } \\ \text { Central Calif Sta H } & 3580 & 1.50 & 125 & 0.062 & \text { (Reimers et al., 1992) } \\ \text { East N. Pac NH 18 } & 146 & 0.30 & 106 & 0.530 & \text { (Devol and Christensen, 1993) } \\ \text { Central Calif Sta F } & 2000 & 1.10 & 75 & 0.056 & \text { (Reimers et al., 1992) } \\ \text { Central Calif Sta L } & 1890 & 0.90 & 72 & 0.053 & \text { (Reimers et al., 1992) } \\ \text { San Clemente Basin } & 1900 & 0.80 & 58 & 0.045 & \text { (Bender et al., 1989) } \\ \text { Borderland Basins-SCI } & 2053 & 0.70 & 58 & 0.040 & \text { (Berelson et al., 1996) } \\ \text { Central Calif Sta E } & 1570 & 0.80 & 50 & 0.048 & \text { (Reimers et al., 1992) } \\ \text { Central Calif Sta D } & 1440 & 1.00 & 33 & 0.035 & \text { (Reimers et al., 1992) } \\ \text { Central Calif Sta C } & 1200 & 0.40 & 26 & 0.048 & \text { (Reimers et al., 1992) } \\ \text { Central Calif Sta K } & 1000 & 0.50 & 20 & 0.043 & \text { (Reimers et al., 1992) } \\ \text { Central Calif Sta A } & 585 & 0.30 & 17 & 0.050 & \text { (Reimers et al., 1992) }\end{array}$


Aller, R. C., Blair, N. E., and Rude, P. D.: Remineralization rates, recycling, and storage of carbon in Amazon shelf sediments, Cont Shelf Res, 16, 753-786, 1996.

Bender, M., Jahnke, R., Weiss, R., Martin, W., Heggie, D. T., Orchardo, J., and Sowers, T.: Organic arbon oxidation and benthic nitrogen and silica dynamics in San Clemente Basin, a continental borderland site, Geochmica et Cosmochimica Acta, 53, 685-697, 1989.

Berelson, W. M., McManus, J., Coale, K. H., Johnson, K. S., Kilgore, T., Burdige, D., and Pilskaln, C.: Biogenic matter diagenesis on the sea floor: A comparison between two continental margin transects., J Mar Res, 54, 731-762, 1996.

Canfield, D. E.: Sulfate reduction and oxic respiration in marine sediments: implications for organic carbon preservation in euxinic environments, Deep-Sea Research, 36, 121-138, 1989.

Canfield, D. E., Jørgensen, B. B., Fossing, H., Glud, R., Gundersen, J., Ramsing, N. B., Thamdrup, B., Hansen, J. W., Nielsen, L. P., and Hall, P. O. J.: Pathways of organic carbon oxidation in three continental margin sediments, Mar Geol, 113, 27-40, 1993.

Chanton, J. P., Martens, C. S., and Goldhaber, M. B.: Biogeochemical cycling in an organic-rich coastal marine basin. 8. A sulfur isotopic budget balanced by differential diffusion across the sediment-water interface, Geochim Cosmochim Ac, 51, 1201-1208, 1987.

Devol, A. H. and Christensen, J. P.: Benthic fluxes and nitrogen cycling in sediments of the continental margin of the eastern North Pacific, Journal of Marine Research, 51, 345-372, 1993.

Glud, R. N., Gundersen, J. K., Jørgensen, B. B., Revsbech, N. P., and Schultz, H. D.: Diffusive and total oxygen uptake of deep-sea sediments in the eastern South Atlantic Ocean: in situ and laboratory measurements, Deep-Sea Reserch I, 41, 1767-1788, 1994.

Glud, R. N., Holby, O., Hoffmann, F., and Canfield, D. E.: Benthic mineralization and exchange in Arctic sediments (Svalbard, Norway), Marine Ecology-Progress Series, 173, 237-251, 1998.

Hammond, D. E., McManus, J., Berelson, W. M., Kilgore, T. E., and Pope, R. H.: Early diagenesis of organic material in equatorial Pacific sediments: stoichiometry and kinetics, Deep-sea research II, 43, 1365-1412, 1996.

Hulth, S., Blackburn, T. H., and Hall, P. O. J.: Arctic sediments (Svalbard): consumption and microdistribution of oxygen, Mar Chem, 46, 293-316, 1994.

Jørgensen, B. B.: Case study-Aahus Bay. In: Eutrophication in Coastal Marine Ecosystems, Jørgensen, B. B. and Richardson, K. (Eds.), American Geophysical Union, Washington DC, 1996.

Jørgensen, B. B. and Revsbech, N. P.: Oxygen uptake, bacterial distribution, and carbon-nitrogen-sulfur cycling in sediments from the Baltic SeaNorth Sea transition, Ophelia, 31, 29-49, 1989.

Lohse, L., Helder, W., Epping, E. H. G., and Balzer, W.: Recycling of organic matter along shelf-slope transect across the N. W. European Continental Margin (Goban Spur), Prog Oceanogr, 42, 77-110, 1998.

Murray, J. W. and Grundmanis, V.: Oxygen consumption in palagic marine sediment, Science, 209, 1527-1530, 1980.

Reimers, C. E.: An in situ microprofiling instrument for measuring interfacial porewater gradients: methods and oxygen profiles from the North Pacific ocean, Deep-sea research, 34, 2019-2035, 1987. 
Reimers, C. E., Fischer, K. M., Merewether, R., Smith, K. L., and Jahnke, R. A.: Oxygen microprofiles measured in situ in deep sea sediments, Nature, 320, 741-744, 1986.

Reimers, C. E., Jahnke, R. A., and McCorkle, D. C.: Carbon fluxes and burial rates over the continental slope and rise off central California with implications for the global carbon cycle, Global Biogeochem Cy, 6, 199-224, 1992.

Reimers, C. E. and Suess, E.: The partitioning of organic carbon fluxes and sedimentary organic matter decomposition rates in the ocean, Mar Chem, 13, 141-168, 1983.

Rysgaard, S., Thamdrup, B., Risgaard-Petersen, N., Fossing, H., Berg, P., Christensen, P. B., and Dalsgaard, T.: Seasonal carbon and nutritient mineralization in a high-Arctic coastal marine sediment, Young Sound, Northeast Greenland, Marine Ecology-Progress Series, 175, 261-276, 1998.

Sagemann, J., Jørgensen, B. B., and Greeff, O.: Temperature dependence and rates of sulfate reduction in cold sediments of Svalbard, Arctic Ocean, Geomicrobiology Journal, 15, 85-100, 1998.

Thamdrup, B. and Canfield, D. E.: Pathways of carbon oxidation in continental margin sediments off central Chile, Limnology and Oceanography, 41, 1629-1650, 1996.

Thamdrup, B., Fossing, H., and Jørgensen, B. B.: Manganese, iron, and sulfur cycling in a coastal marine sediment, Aarhus Bay, Denmark, Geochim Cosmochim Ac, 58, 5115-5129, 1994. 